\title{
Towards the Integration of Process and Quality Control using Multi-agent Technology
}

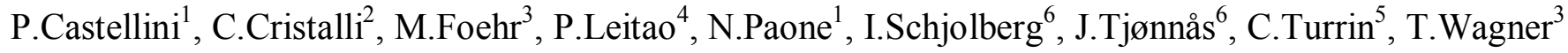 \\ ${ }^{1}$ Università Politecnica delle Marche, Dipartimento di Meccanica, Ancona (AN), Italy, n.paone@univpm.it \\ 2 AEA, Angeli di Rosora (AN), Italy, c.cristalli@loccioni.com \\ ${ }^{3}$ Siemens AG, Corporate Technology, Global Technology Field Project Engineering, Erlangen, Germany, \{thomaswagner, \\ matthias.foehr\}@siemens.com \\ ${ }^{4}$ Polytechnic Institute of Bragança, Campus Sta Apolónia, Apartado 1134, 5301-857 Bragança, Portugal, pleitao@ipb.pt \\ ${ }^{5}$ Whirlpool Europe, Cassinetta di Biandronno (VA), Italy, Claudio_Turrin@whirlpool.com \\ ${ }^{6}$ SINTEF-ICT, N-7465 Trondheim, Norway, Ingrid.Schjolberg@sintef.no
}

\begin{abstract}
The paper introduces a vision on the design of distributed manufacturing control systems using the multi-agent principles to enhance the integration of the production and quality control processes. It is highlighted how agent technology may enforce interaction of manufacturing execution system and distributed control system, enhancing the exploitation of the available information at the quality control and process control levels. A specific focus is made on a suitable engineering methodology for the design and realization of such concept. Innovation is also presented at the level of adaptive process control and self-optimizing quality control, with examples related to a home appliance production line.
\end{abstract}

\section{INTRODUCTION}

European research aims to develop innovative production systems driven by economical and societal factors to transform EU industry from a resource intensive to a sustainable knowledge-based industrial environment. Many strategic foresight studies at international level highlight the shift from competition by reducing costs towards competition by added value [1], [2]. A number of different drivers play the role of enabling factors for this new manufacturing reality; among these, the strategic research agenda made by the Manufuture European Technology Platform [3] points out the need for enabling technologies, oriented to flexible and intelligent processes, and new concepts for process control and sensor technology. A relevant current research is focused on the development of adaptive production systems that overcome existing process limitations and enable new manufacturing and processing methods. The trend is towards Decentralized Manufacturing Systems (DMS) which allow process control at different factory levels.

The objective of the GRACE (InteGration of pRocess and quAlity Control using multi-agEnt technology) project [4] is to study, conceive, develop, implement and validate a collaborative Multi-agent System (MAS) which operates at all stages of a production line, integrating process control with quality control at local and global level. The project will establish a multi-agent architecture, encountering a large variety of disturbances and changes in process parameters and variables. This approach is in line with the current trend to build modular, intelligent and distributed control systems.

The choice of the multi-agent systems paradigm [5] is motivated by its intrinsic characteristics, namely the decentralization of control over distributed autonomous and cooperative entities, which is particularly suited to implement control at factory level, to achieve modularity, flexibility, robustness and adaptation. This paradigm has the capability to respond promptly and correctly to change, and differs from the conventional approaches due to its inherent capabilities to adapt to emergence without external intervention [5].

In order to improve reliability of diagnosis of quality control systems, these systems are designed to embody selfoptimization to keep measurement uncertainty under control. Adaptive behaviour of production processes at local and global level complements the system design.

The objective of this paper is to describe the main principles of the GRACE vision, presented in section II, and its scientific contributions. Section III focuses on the engineering methodology for DMS based on the GRACE multi-agent system platform and section IV introduces the expected contribution and innovation. At last, section $\mathrm{V}$ rounds up the paper with the conclusions.

\section{THE GRACE VISION}

The purpose of the GRACE project is the design of distributed manufacturing control systems for production using the MAS principles and integrating the production and quality control processes. The GRACE system will act at the Manufacturing Execution System (MES) level of the automation pyramid, as illustrated in Fig. 1, interacting with the lower-control level distributed control system (DCS) which is related to the control of the automation devices, e.g., by using a network of programmable logic controllers (PLC). Agent technology enforces the interaction of MES and DCS, enhancing the exploitation of available information at the quality control and process control levels. These concepts are demonstrated for a home appliance production system, particularly for washing machines. 


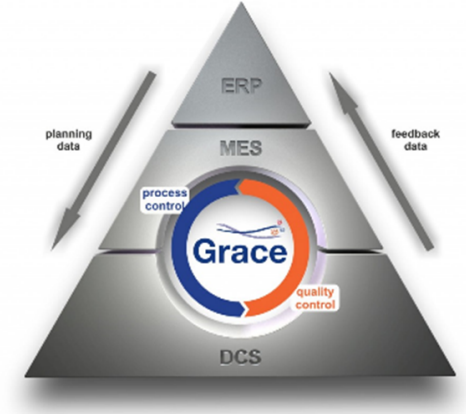

Fig. 1. DCS-MES focus of GRACE Project in the Automation Pyramid.

The processing and the quality control stations of a home appliance production line are treated as intelligent agents, and the whole production process is supervised and controlled through the integrated and coordinated operation of a network of collaborative individual agents, each one with its own objectives and behaviours, possessing its own perceptive and cognitive capabilities. The proposed architecture is inspired in the principles established in previous manufacturing multiagent systems architectures, namely PROSA [6], ADACOR [7] and PABADIS-PROMISE [8], adapted according to the particularities of the on-demand production process.

In such distributed environment, several types of agents are identified according to the process to control and to their specialization, as illustrated in Fig. 2: product type agents (PTA), product agents (PA), resource agents (RA), quality control agents (QCA) and independent meta agents (IMA).

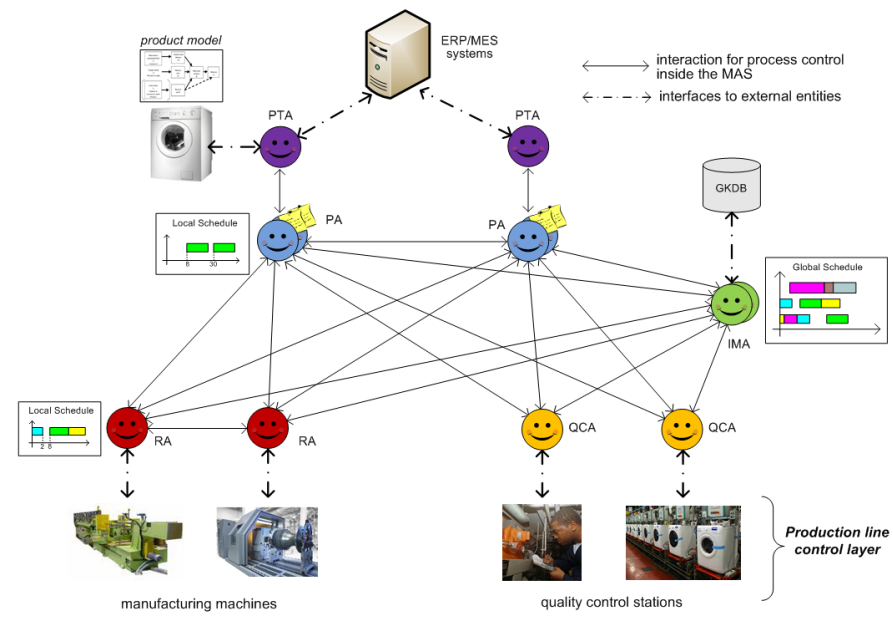

Fig. 2. Multi-agent System Architecture for the Production Line.

The product type agents represent the catalogue of products that can be produced by the factory plant and contain the process and product knowledge required to produce the product, namely the product structure and the process plan. These agents are responsible for monitoring the historical product execution and for adapting/optimizing the process plan according to the feedback of previous executions.

The product agents manage the production of product instances in the factory plant. They possess a process plan to produce the product and interact with the RA agents for the process control and with the QCA agents for the quality control. They are responsible for the product process management, the re-routing of pallets, the production data collection, and the adaptation in the selection of production and quality control programs/components/parameters.

The resource agents are associated to the physical equipments of the production line, such as robots and machines, and manage the execution of their operations. These agents are responsible for the dispatching and process control, resource monitoring and optimization/adaptation of the operation parameters. The RA agents may also represent the manual operations performed by human operators.

The quality control agents are associated to the quality control stations and manage the execution of quality control operations in the production line. These agents are mainly responsible to implement the testing/quality control functions on the parts being manufactured and on the final product in the factory plant, and to adjust the quality control algorithms and parameters, based on the environmental conditions and information about previous executed operations. The results provided by the QCA agents allow closing the control loops, making available reliable information to be used by other agents for adaptation of factory processes at global level.

The independent meta agents introduce a kind of hierarchy in the decentralized system, allowing the implementation of global supervisory control and optimized decision-making mechanisms, e.g., defining and adapting global policies for the system. They collect data from individual agents, and identify meaningful correlations that are then suggested as new policies to the individual processes. These agents will monitor data over time, perform trend analysis and create models in order to update reference values of particular thresholds. Additionally, these agents feed data to the global knowledge database of the production system.

The interaction among agents, necessary for the emergence of an overall distributed control system, are designed to enhance integration and collaboration, opening a sound perspective for:

- Developing self-adaptability to desired variation of process set-point and process variables and to unplanned fluctuations of process/product parameters.

- Developing adaptive procedures and self-learning for quality control systems at local and global level.

- Developing supervisory control schemes at factory level which maximize production efficiency and product quality.

- Developing factory-level decision-making strategies based on data analysis methods exploiting information from all current processes and form historic data base.

The interaction between agents requires that agents can understand themselves, using interaction protocols, a proper agent communication language and ontologies. Particularly, the use of ontologies assumes a crucial role for a common understanding on the vocabulary used during the exchange of knowledge, supporting the collaborative and reconfiguration processes. For this purpose, an ontology to be used in home appliances production lines will be defined, considering the 
production and quality control knowledge, aiming to overcome the interoperability problem.

One peculiarity of this project is to consider adaptability and self-learning at different levels in various processes. While these concepts are generally linked to process control in manufacturing and assembly processes, this project will also explore their implementation and impact within the measurement processes in charge of quality control, so that the measurement system itself will adapt to the variable characteristics of the object under test, in order to minimize uncertainty and provide reliable information supporting process control and product diagnosis. Furthermore, adaptation will also be implemented on the final products. In this way, a proper set of control parameters is adjusted and stored in each controller that manages the functions of the product once in use, in order to keep its performance within tight specifications (especially energy efficiency and vibroacoustic emission) even in case of dispersion of characteristics among different individual products, due to natural tolerances of production processes or to problems occurred during assembly. This allows to significantly reduce dispersion of performance among individual products, taking into account the results of the tests performed during the assembly and at the end-of-line.

\section{ENGINEERING Methodology}

Within the GRACE project a new engineering methodology for DMS based on the GRACE MAS platform will be developed, creating suitable and effective engineering concepts for decentralized automation, and to render the new GRACE control concepts on industrial application scenarios. Hence, the engineering methodology aims for a broad public acceptance of the GRACE project by providing a kind of user manual for the engineering of multi agent systems.

As mentioned in section II, one research approach to enable flexibility and adaptability of a production system is the use of DMS controlled by agents [7], [8]. In parallel to these research activities, the mechatronic concept has been developed to a main research focus within international engineering approaches [9], [10].

The GRACE engineering methodology combines both approaches by defining a mechatronic oriented engineering process where the mechatronic units build a production system controlled by RA and QCA agents. One main focus by developing this methodology will be the influence of the plant engineering on the final product quality, as illustrated in the causal chain of Fig. 3:
1. The aim of the engineering process is to design a manufacturing system (including the engineering of the production process and the technological structure of the manufacturing system), which produces the product according to the specifications given by the product design. The engineering of the production system influences the properties of the production system by defining the production process, manufacturing steps and the production stations. For this reason it defines how accurate the product specifications can be realized.

2. The manufacturing system is producing the products. So, the process and quality related properties of the manufacturing system are directly influencing the product properties. During the production process even more external influences exist, e.g. the quality of purchased parts or the quality of the used tooling equipments are relevant for the reachable product quality. They cannot be influenced by engineering.

3. In the same way a product consists of several components, the quality of a product is defined by the properties of its single components and by the quality of the combination of the single parts. These dependencies between the product quality features and the product properties can be identified easily by using the "House of Quality" approach [11]. It reflects the relations between the technical engineering of a product and the customer requirements regarding a product and therefore gives a mapping of product quality features to product properties.

4. In case of changed/new customer requirements or nonfulfillment of product quality, the product has to be modified or changed. This includes changes of the product properties of several components. These related properties have to be analyzed by retrojecting the product quality features to product properties. Therefore also the House of Quality gives a methodic approach.

5. Whether modifications in the product design or in the manufacturing process are necessary, such modifications usually require changes in the manufacturing system. If the product properties, which have to be modified, are identified, the manufacturing stations influencing them can be analyzed.

6. If any changes in the production system are necessary, modifications of single manufacturing stations adding new manufacturing stations or a variation of the production process might be needed. These necessary changes in the manufacturing system also influence the engineering process.

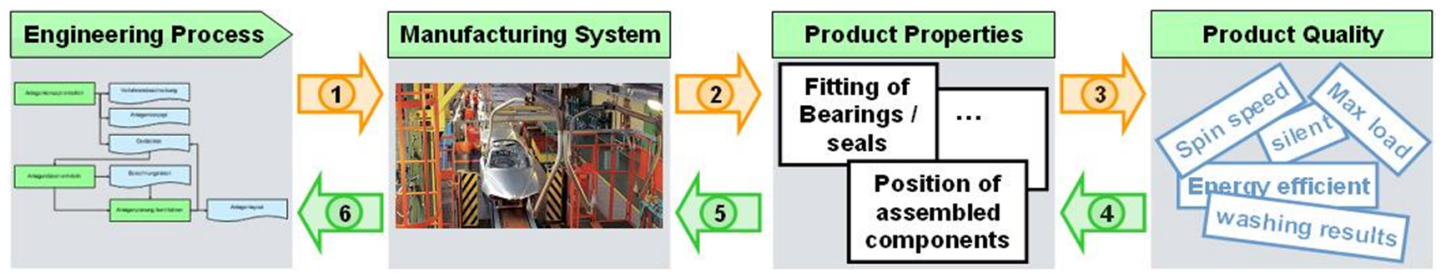

Fig. 3 Cycle of Engineering $\rightarrow$ Quality influences. 
Within the development of the GRACE engineering methodology these steps/dependencies are being analyzed. By setting up common workshops and using the industrial application experience of the project partners the industrial applicability of GRACE will be ensured.

\section{CONTRIBUTION AND INNOVATION}

\section{A. Innovation at the level of process control}

One of the project goals is to develop methods for the automatic adjustment of parameters of the machines operating along the production line, and in addition to suggest new and more robust global feedback loops to improve the production process and the product quality. Basic control theory will be applied to ensure stability and robustness both on a local and global level. Moreover, optimization in network of systems by use of decomposition methods as proposed in [12] and demonstrated in [13] will be considered. The research contribution and innovation lies in the design and implementation of the above methods in a real factory environment. Increased performance of the production system as well as increased product quality will be shown.

Resources are often controlled by rigid PID controllers usually supplied by the machine supplier. Such controllers may not be sufficient to handle variations and disturbances. Both local and global adaptive and self-tuning controllers are in focus as they are flexible with respect to choice of the methodology for the controller design and for the process identification scheme. In addition, self-optimization techniques will be considered as a method for optimal control structure design to select controlled variables in a way that unknown disturbances result in minimum operational loss. Self-organisation, which is a property where entities reorganise based on an optimization criteria, will be considered on a global level.

A simple simulation example is presented as preliminary result to illustrate the effect of self-organization, and local and global adaptive control in a typical process: the automatic assembly of two pieces by insertion of one into the other. Several parameters could affect the quality of the operation as variations in geometry, temperature and stiffness in the material. The bearing insertion, illustrated in Fig. 4, is an example of such a process.

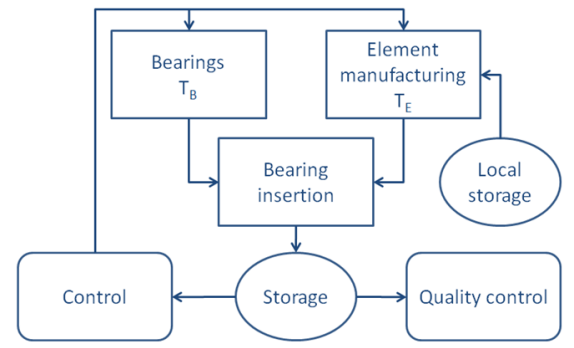

Fig. 4 Bearing Insertion, Quality Control and Temperature Adaption.

To increase the quality of the process, an adaptive temperature controller is implemented, and the temperature variations in the elements are taken into account. Each of the components has a temperature property depending on if they are stored inside or outside the factory plant. As a consequence the initial temperature of the two components may be different. To avoid temperature variations, elements may have to rest at a small local storage inside the factory to stabilize the temperature and thereby improve the quality of the process. The main purpose of the adaptive controller is to estimate the minimum required storage time and in this way the integration of process and quality control is achieved.

It is assumed that each product has a quality measure $q_{m}$. If this measure is outside an acceptance interval $\left\{q_{\text {min }}, 0\right.$, $\left.q_{\max }\right\}$ the product is rejected and removed from the end storage. The quality parameter is a difference function of the temperature of two components. This is given by:

$$
q_{m}=T_{B}-T_{E}+u
$$

where $T_{B}$ and $T_{E}$ are the temperatures of the bearing and element, and $u$ represents the measurement disturbance and the process uncertainty.

The temperature dissipation equation for the element is a simple first order differential equation with the solution given by: $\quad T_{E}(t)=T_{f}+\left(T_{E}(0)-T_{f}\right) e^{-k t}$

where $T_{E}(0)$ is the temperature of the element when the element is ready for the insertion process, $T f$ is the air temperature of the factory and $k$ is the heat coefficient. It is assumed that the temperature of the bearing is equal to the air temperature. Based on this solution and a minimum temperature difference threshold, the minimum storage time $\Delta t$ can be calculated and is given by the following equation:

$$
\Delta \mathrm{t}>\left(-\ln \left(\left|\mathrm{T}_{\mathrm{E}}-\mathrm{T}_{\mathrm{f}}\right|\right)-\ln \left|\mathrm{T}_{\mathrm{E}}(0)-\mathrm{T}_{\mathrm{f}}\right|\right) / \hat{\mathrm{k}}
$$

As can be seen from the minimum storage time estimate, an estimate of the heat coefficient $k$ is needed. An adaptive law will provide this estimate and an estimate of $q_{m}$ :

$$
\begin{gathered}
\dot{\hat{T}}_{E \dot{\hat{k}}}=\hat{k}\left(T_{B}-\widehat{T}_{E}\right) \\
\tilde{\tilde{q}}:=q_{m}-\hat{q} \tilde{q} \\
\hat{q}:=T_{B}-\widehat{T}_{E}
\end{gathered}
$$

where $\Gamma$ is the adaptive controller gain.

Provided no noise and no disturbance affect the quality measure, the adaptive law presented above makes the equilibrium of the respective system uniformly exponential stable. If noise is bounded, the solution of the system is uniformly bounded. This can be proven by using a proper Lyapunov function. Simulations are performed to compare three different scenarios, each representing different control schemes:

1. Storage feedback with feed forward from the demand. The inventory level at the cool-off storage is also controlled at a reference.

2. Self-organisation of resources feed forward from demand. Resources can vary between different processes and can be re-organized based on a simple global optimization algorithm.

3. Adaptive control with feed forward from demand and self-organization of resources. Uses the minimum storage time control derived in the previous section instead of level. 
The simulation, see Table I, shows that the trash is significantly reduced by introducing local and global adaptive control loops as well as self-organizing properties.

\begin{tabular}{|c|c|c|c|c|c|}
\hline TABLE I: SIMULATION RESULTS, NUMBER OF PRODUCTS \\
\hline Scenario & Produced & Delivered & Trash & Stored & $\begin{array}{c}\text { Cold } \\
\text { Storage }\end{array}$ \\
\hline 1 & 460 & 304 & 125 & 30 & 9 \\
\hline 2 & 480 & 334 & 115 & 30 & 10 \\
\hline 3 & 415 & 350 & 34 & 30 & 10 \\
\hline
\end{tabular}

Similar control strategies will be developed and tested by simulations on other appliance processes as well as on a global level. The succeeding step is the implementation in an appliance factory.

\section{B. Innovation at the level of measurement systems}

Quality control systems perform industrial diagnostics, a procedure to detect defects of a product under test. Once the defect is defined, a set of physical quantities is measured under controlled conditions for each product and features are computed and compared to reference values for diagnosis.

Therefore quality control means measuring the conformity to specifications. Any quality control system is a source of quantitative information to be managed by agents, which can be used locally to perform the quality control task, that can also be stored in a data base for future retrieval and use, or that can be fed backward or forward to the production processes and used for process control.

The agents responsible to manage quality control are designed for sharing information and possibly closing control loops with agents responsible to manage the resource process activities and to provide reliable information for adaptation of factory processes at global level.

Quality controls rely on quantitative measurements and this brings to the issue of measurement uncertainty [16]. False positives or false negatives at diagnostic level depend on measurement uncertainty at sensor level and any following decision/action taken on the basis of measured data will be affected by measurement uncertainty [15], [18]. Therefore measurement uncertainty becomes the main aspect to be dealt with in the development of quality control systems.

An innovative approach in the GRACE project is the development of an active management of measurement uncertainty, so to realize a self-optimizing measurement system. Any measurement system outputs a signal $\mathrm{q}_{\mathrm{o}}$; ; this information is related to the input quantity $\mathrm{q}_{i}$ by the calibration function $\mathrm{q}_{\mathrm{o}}=\mathrm{f}\left(\mathrm{q}_{\mathrm{i}}, \mathrm{q}_{\mathrm{Ik}}, \mathrm{q}_{\mathrm{dj}}\right), k=1, \ldots . M, j=1, \ldots . N$, where $\mathrm{q}_{\mathrm{Ik}}$ are the influencing parameters and $\mathrm{q}_{\mathrm{dj}}$ the disturbances, which can be classified in interfering and modifying disturbances. They act on the system independently from the input quantity $\mathrm{q}_{\mathrm{i}}$, and therefore determine measurement uncertainty $U[17]$.

The block-diagram of Fig. 5 represents the innovative concept of self-optimization at the level of measurement system. It consists in controlling one or more influencing parameters $\mathrm{q}_{\mathrm{I} j}$, so to keep signal quality at a desired level. By signal quality $Q_{P}$ we mean a physical quantity which is known to be correlated to measurement uncertainty $U$; maximizing $Q_{P}$ allows to reduce $U$. In most measurement systems, it can be identified an influencing parameter $\mathrm{q}_{\mathrm{Ik}}$ which may be kept under control. Generally signal quality $Q_{P}$ exhibits a maximum at some value of $\mathrm{q}_{\mathrm{Ik}}$. Such an active management of uncertainty involves implementation of hardware and software tools for real time control of an influencing parameter of the measurement system.

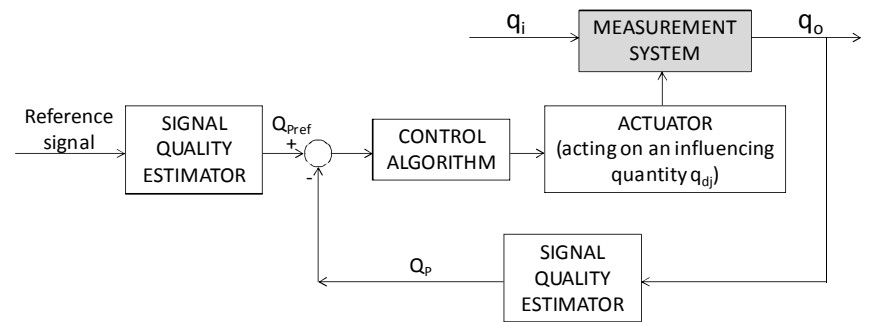

Fig. 5 Self-optimization at the Level of Measurement System.

Fig. 6 reports results in the example of a laser vibrometer used in a quality control station for mechanical fault detection. Here the quality parameter $Q_{P}$ is the nondimensional amplitude of the optical interference signal before demodulation (values between 0 and 1). When it decreases, the output signal of the vibrometer is noisy [18], [19] and uncertainty $U$ increases. Signal quality of a laser vibrometer can be optimized by small displacement of the laser beam on the surface. The control loop of Fig. 5 performs such a task on the influencing parameter beam position. Another example of industrial interest is a vision system used for measurement; in this case, signal quality parameter $Q_{P}$ [20] is the image contrast, and the influencing parameter is illumination. The control loop therefore controls illumination.

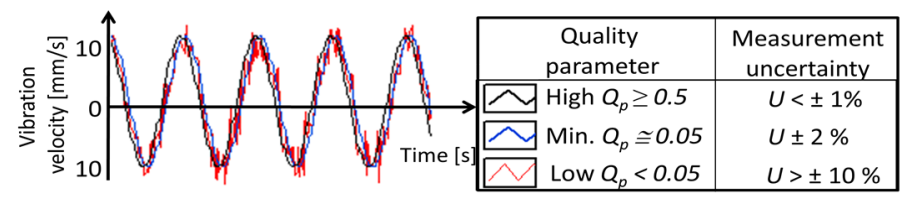

Fig. 6 Dependence of Measurement Uncertainty U on Signal Quality $Q_{P}$ in Laser Doppler Vibration Measurements.

Uncertainty $U$ once estimated and associated to its confidence level, can be optimized for the given scenario. Different strategies are to determine:

1. Minimum $U$ given the external conditions.

2. Minimum $U$ compatible with time available for test.

3. Maximum $U$ compatible with "distance" between current estimate of the actual measured value and threshold level used for diagnosis.

$U$ is then associated to a cost function which weights the cost necessary to achieve the desired level (e.g. averaging of signals may improve $U$ but requires more time for the measurement; time is a cost).

Finally, the achieved uncertainty and its confidence level is provided to other agents, so that any decision within the MAS is weighted by the confidence level of the available 
information, thus providing a quantitative estimate on its reliability. This enhances the value of any decision based on measurement.

\section{Innovation at the level of MAS}

The resulting MAS architecture is based on a society of autonomous agents co-operating along the home appliance production line, integrating the production and quality control processes, and guaranteeing the achievement of:

1. Modularity and flexibility, i.e. plugging in or out autonomous components/agents.

2. Run-time adaptation, i.e. applying local self-adaptive and self-organization concepts to adapt the system behaviour according to the unplanned changes.

3. Run-time and on the fly reconfiguration, i.e. adding, removing or changing components without the need to stop, re-program and re-initialise the other components.

4. Robustness and responsiveness, i.e. a better response to changes/failures, by using distributed control structures.

The interaction patterns among agents are designed to enhance integration and collaboration among intelligent units, to perform the re-configuration, adaptation and optimization in the production and quality control processes. Some concepts inherited from the theory of complexity, emergence behaviour and self-organization will be considered in the design of interaction patterns that require a more complex behaviour, namely the re-routing of pallets to face the occurrence of unexpected scenarios.

An innovative aspect of the GRACE project at the multiagent systems level is related to the fully assessment of the multi-agent systems benefits in real industrial environments. In fact, there is a gap today between theory on development of adaptive multi-agent systems, and their implementation in manufacturing industry [21], [22]. The GRACE project seeks to fill this gap, by demonstrating the effective applicability of multi-agent systems in a real world case, considering the case of a factory operating with a production on-demand, where some changes of process set-point and of product characteristics can be predictable, and other random unplanned changes of process variables may always occur.

\section{CONCLUSIONS}

This paper highlights the vision and main principles of the GRACE project that aims to develop a MAS system to operate in a production line integrating production and quality control. The paper presents self-adaptation and selfoptimization at level of production and quality control processes, at local and global levels, which enforce the capability to provide more flexibility, robustness and responsiveness. An engineering methodology for the development of DMS based on the GRACE MAS principles will contribute for a broad public acceptance of the GRACE results in the industrial domain and will enforce its impact.
The GRACE project is in an initial phase and future work is related to the development and implementation of the described GRACE vision.

\section{ACKNOWLEDGMENT}

This work has been partly financed by the EU Commission, within the research contract GRACE coordinated by Univ. Politecnica delle Marche and having partners SINTEF, AEA srl, Instituto Politecnico de Bragança, Whirlpool Europe srl, Siemens AG.

\section{REFERENCES}

[1] Committee on Visionary Manufacturing, "Visionary Manufacturing Challenges for 2020", National Academic Press, US, 1998.

[2] EC, "ManuFuture, A Vision for 2020", Report of the High-level Group, Brussels, European Commission, 2004.

[3] MANUFUTURE-Strategic Research Agenda Manufuture - 2006

[4] GRACE project web site, http://grace-project.org/.

[5] Wooldridge M., "An Introduction to Multi-Agent Systems", John Wiley \& Sons, 2002.

[6] Brussel, H. V., Wyns, J., Valckenaers, P., Bongaerts, L., "Reference Architecture for Holonic Manufacturing Systems: PROSA", Computers in Industry, 37(3), pp. 255-274, 1998.

[7] Leitao, P., Restivo, F., "ADACOR: An Agile and Adaptive Holonic Architecture for Manufacturing Control", Computers in Industry, 57(2), pp. 121-130, 2006.

[8] PABADIS'PROMISE consortium, "Industrial Application of the PABADIS'PROMISE System - White Paper", October 2008.

[9] VDI2206, "Design Methodology for Mechatronic Systems", Association of German Engineers, Beuth, Berlin, 2004.

[10] Lüder, A., Hundt, L., Foehr, M., Holm, T., Wagner, T., Zaddach, J.-J., "Manufacturing System Engineering with Mechatronical Units", Proceedings of the IEEE Conference on Emerging Technologies \& Factory Automation, pp. 1-8, 2010.

[11] Hammer, E., "House of Quality (HoQ)", 2011 (available at http://www-classic.uni-graz.at/inmwww/NEU/lehre/pdf/ Hammer_House_of_Quality.pdf).

[12] Kercelli, L., Sezer, A., Ôztoprak,F., Yolum, P., Birbil, S, "MANGO: A Multiagent Environment for Global Optimization", Proceedings of the $7^{\text {th }}$ Conference on Autonomous and Multi-agent Systems, 2008.

[13] Holmgren, J., Persson, J., Davidsson, P., “Agent based Decomposition of Optimization Problems", Proceedings of the $1^{\text {st }}$ International Workshop on: Optimisation in Multi-Agent Systems, 2008.

[14] ISO/IEC Guide 98-3:2008 - Uncertainty of Measurement - Part 3: Guide to the Expression of Uncertainty in Measurement (GUM:1995).

[15] Rossi, G.B., Crenna, F., "A Probabilistic Approach to Measurementbased Decisions", Measurement, 39, pp.101-119, 2006.

[16] Eidukeviciute, M., Volkovas, V., "Measurement Uncertainty in Vibromonitoring Systems and Diagnostic Reliability Evaluation", Journal of Sound and Vibration, vol. 308, pp. 625-631, 2007.

[17] Doebelin, E., "Measurement Systems: Application and Design", Mc Graw-Hill, 1990.

[18] Martin, P., Rothberg, S., "Introducing Speckle Noise Maps for Laser Vibrometry", J. Optics and Lasers in Engineering, vol.47, pp. 431442, 2009.

[19] Agostinelli, G., Cristalli, C.,Paone, N., Serafini, S., "Drop-out Noise of Laser Vibrometers Measuring on Varnished Steel Surfaces of Appliance Cabinets for Industrial Diagnostics", Proc. of the $9^{\text {th }}$ Int. Conf. on Vibration Measurements by Laser and Noncontact Techniques, 2010

[20] Marchant, J.A., "Testing a Measure of Image Quality for Acquisition Control", Image and Vision Computing, vol. 20, pp.449-458, 2002.

[21] Leitão, P., “Agent-based Distributed Manufacturing Control: A Stateof-the-art Survey", Engineering Applications of Artificial Intelligence, 22(7), pp. 979-991, 2009.

[22] Pechoucek, M., Marik, V., "Industrial Deployment of Multi-agent Technologies: Review and Selected Case Studies", Autonomous Agents and Multi-agent Systems, 17(13), pp. 397-431, 2008. 